\title{
COBALT: Development of a Platform to Flight Test Lander GN\&C Technologies on Suborbital Rockets
}

\author{
John M. Carson III ${ }^{1,3, *}$, Carl R. Seubert ${ }^{3, \dagger}$, Farzin Amzajerdian ${ }^{2, \ddagger}$, Chuck Bergh ${ }^{3, \S}$, \\ Ara Kourchians $^{3, \diamond}$, Carolina I. Restrepo ${ }^{1, \nabla}$, Carlos Y. Villalpando ${ }^{3, \uparrow}$, Travis V. O’Neal ${ }^{4, \star}$, \\ Edward A. Robertson ${ }^{1, \|}$, Diego F. Pierrottet ${ }^{5, * *}$, Glenn D. Hines ${ }^{2, \dagger \dagger}$, Reuben Garcia ${ }^{4, \ddagger}$ \\ ${ }^{1}$ NASA Johnson Space Center (JSC), $\quad{ }^{2}$ NASA Langley Research Center (LaRC), \\ ${ }^{3}$ Jet Propulsion Laboratory (JPL), California Institute of Technology, \\ ${ }^{4}$ Masten Space Systems (MSS), ${ }^{5}$ Coherent Applications, Inc. (CAI)
}

\begin{abstract}
The NASA COBALT Project (CoOperative Blending of Autonomous Landing Technologies) is developing and integrating new precision-landing Guidance, Navigation and Control (GN\&C) technologies, along with developing a terrestrial flight-test platform for Technology Readiness Level (TRL) maturation. The current technologies include a thirdgeneration Navigation Doppler Lidar (NDL) sensor for ultra-precise velocity and lineof-site (LOS) range measurements, and the Lander Vision System (LVS) that provides passive-optical Terrain Relative Navigation (TRN) estimates of map-relative position. The COBALT platform is self contained and includes the NDL and LVS sensors, blending filter, a custom compute element, power unit, and communication system. The platform incorporates a structural frame that has been designed to integrate with the payload frame onboard the new Masten Xodiac vertical take-off, vertical landing (VTVL) terrestrial rocket vehicle. Ground integration and testing is underway, and terrestrial flight testing onboard Xodiac is planned for 2017 with two flight campaigns: one open-loop and one closed-loop.
\end{abstract}

\section{Introduction}

The COBALT Project was initiated in 2016 to continue the development, maturation and terrestrial flight testing of NASA precision-landing Guidance, Navigation and Control (GN\&C) technologies. ${ }^{1}$ The project is leveraging technologies and capabilities that have been in work for more than a decade across the agency through prior projects such as ALHAT (Autonomous precision Landing and Hazard Avoidance Technology $)^{2-7}$ and ADAPT (Autonomous Descent and Ascent Powered-Flight Testbed), ${ }^{8,9}$ as well as through current projects developing the JPL Lander Vision System (LVS) ${ }^{10-12}$ (to be infused onto the Mars 2020 mission) and the LaRC Navigation Doppler Lidar (NDL). ${ }^{13-16}$ COBALT is developing a new generation of NDL, which provides ultra-precise LOS velocity plus range measurements, along with a terrestrial test platform that integrates the NDL and LVS into a self-contained payload for flight testing. The payload will be integrated onto the Masten Xodiac VTVL vehicle for flight testing in 2017.

*COBALT PM, JSC-IPA, AIAA Associate Fellow.

${ }^{\dagger}$ COBALT SE\&I, JPL Team Lead, AIAA Senior Member.

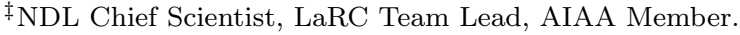

$\S$ COBALT Payload System Engineer.

${ }^{\diamond}$ COBALT Payload Dev \& Test Engineer.

${ }^{\nabla}$ COBALT Deputy PM.

\footnotetext{
ฯ COBALT Software and Avionics Development.

^ Xodiac \& MSS Chief Engineer.

$\|$ COBALT Chief Engineer.

**NDL Systems Engineer.

${ }^{\dagger}$ NDL Chief Engineer.

$\ddagger \ddagger$ MSS Director of Technical Operations.
} 
GN\&C technologies for Precision Landing and Hazard Avoidance (PL\&HA) have been identified by both NASA and National Research Council (NRC) as high-priority capabilities for future robotic and human landing missions. ${ }^{17,18}$ The PL\&HA capabilities enable safe and precise landing at locations too risky for current GN\&C capabilities; such regions include topographically diverse terrain with lander-sized hazards (slopes or rocks), as well regions with pre-positioned surface assets (cached science samples or human-mission infrastructure). Figure 1 illustrates GN\&C landing-system capabilities enabled with current GN\&C sensors and the addition of PL\&HA technologies.

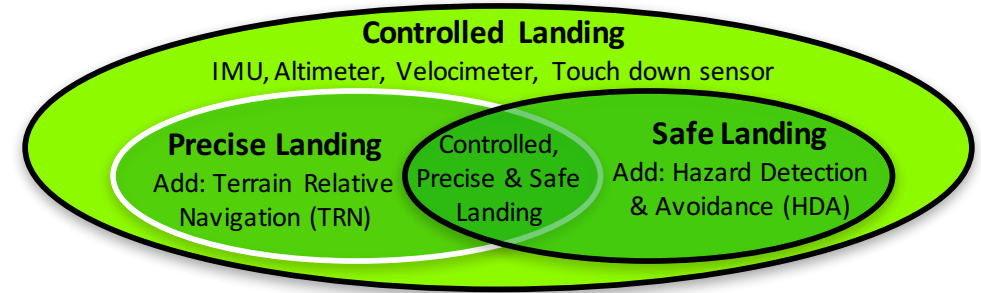

Figure 1. GN\&C landing system capabilities enabled with PL\&HA technologies.

The current state-of-practice GN\&C capabilities within NASA landing missions provide a soft, controlled landing. There is no precise knowledge provided through these capabilities, however, so the landing phase of a mission is blind to the final global landing location (within an a priori assessed landing ellipse) until post-landing data reconstruction. The addition of precision landing through an onboard TRN sensor, such as LVS, and a reconnaissance map provides global position knowledge during descent that can be utilized for onboard, intelligent maneuver planning to minimize landing error. Reconnaissance maps, however, are of finite resolution, and the resolutions are typically not fine enough to a priori detect small terrain features that would pose a hazard to a small, robotic lander. The addition of an onboard Hazard Detection and Avoidance (HDA) system ${ }^{5,6}$ provides local, high-resolution knowledge of the landing terrain during final descent and can be used to implement a small avoidance divert to a safe landing site.

The current COBALT platform integrates LVS and NDL to provide the GN\&C capabilities for precise and controlled soft landing. Future payloads could also incorporate HDA systems. The flight test plans include two campaigns onboard the Masten Xodiac vehicle: one open-loop and one closed-loop. During the open-loop flights, COBALT will be flown as a passive payload for data collection, timing characterization and analysis. While the COBALT estimator provides a navigation solution, Xodiac will navigate with GPS measurements for the open-loop flights. During the closed-loop campaign, will use the COBALT navigation solution throughout the flight to execute the ascent and descent maneuvers to a pre-determined landing location. This paper will provide an overview of the project, as well as the ongoing implementation and testing of the system elements in preparation for the 2017 flight campaigns onboard Xodiac. Further details are also available in a prior COBALT publication. ${ }^{1}$

\section{COBALT Components Overview}

The COBALT platform consists of a sensor assembly, a custom Compute Element (CE) and communication system, a power unit, and the payload frame for integration and testing with the host vehicle (MSS Xodiac). The components of COBALT are illustrated in Figure 2 (left) alongside a photo of the current hardware (right). This configuration leverages components from the prior ADAPT project, ${ }^{8}$ as well as the SURROGATE $^{19}$ and RoboSimian ${ }^{20}$ mobile robot programs. The COBALT payload volume is $0.127 \mathrm{~m}^{3}$ and the target not-to-exceed mass (for Xodiac flights) is $48.5 \mathrm{~kg}$.

The sensor assembly is mounted above the NDL optical head and maintains a $35^{\circ}$ off-nadir pointing angle for the LVS camera (as was used in ADAPT) to avoid seeing the vehicle landing feet in the camera Field of View (FOV). The NDL optical head is mounted so that each of its 3 beams has an equivalent angular rise $\left(25^{\circ}\right)$ from nadir. The overall mounting structure is designed to minimize relative motion between the LVS sensor assembly and the NDL optical head under the vibration loads experienced during flight. 

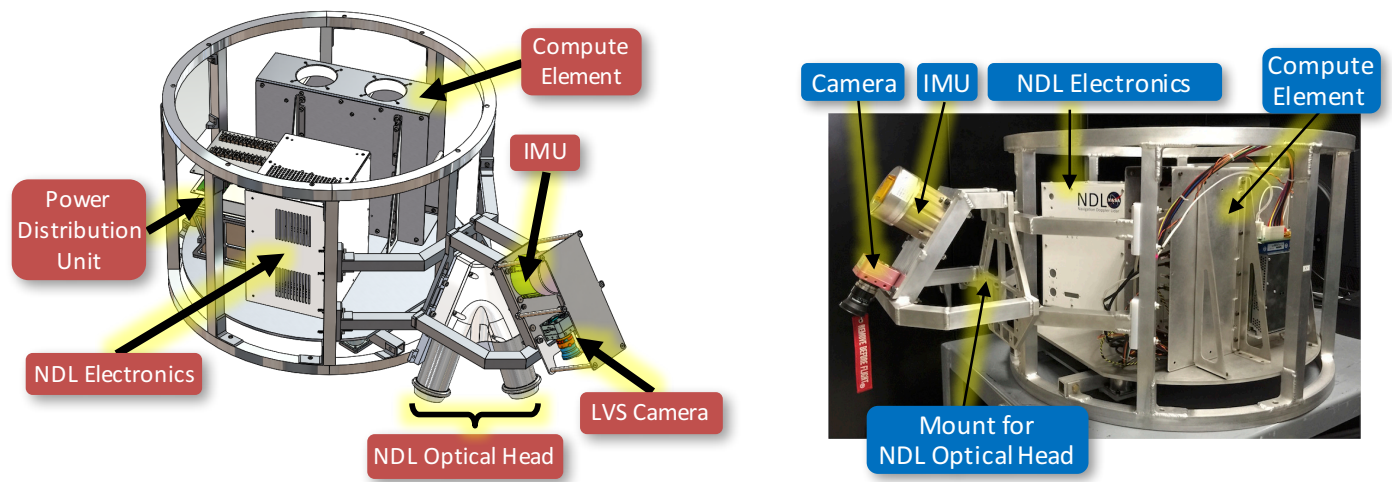

Figure 2. Illustration of COBALT payload (left) and photo of current hardware (right) without NDL optical head.

\section{II.A. Navigation Doppler Lidar}

The COBALT payload incorporates the third-generation (GEN3) NDL for measurements of Line-of-Sight (LOS) velocity and range (See Figure 3). The GEN3 NDL incorporates several design revisions that increase the dynamic performance envelope and reduce the overall size, weight and power of the unit compared to the prototype NDL flown on Morpheus. ${ }^{16}$ The NDL consists of an electronics chassis and a fiber-coupled optical head. The electronic chassis contains all the Lidar components, including transmitter laser, receiver, real-time processor, system controller, and power supply unit. The optical head contains three fiber-coupled lenses and is mounted rigidly to the vehicle with a clear FOV to the ground. The optical head and electronic chassis are con-

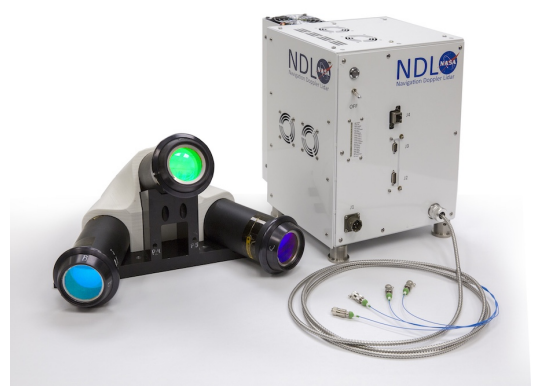

Figure 3. NDL electronics and optical head. nected through a long fiber-optic cable which carries the transmitted beams to the three optical-head lenses and the returned signals, collected with the same lenses, back to the receiver. The GEN3 NDL performance envelope includes a maximum LOS velocity of $200 \mathrm{~m} / \mathrm{s}$ and a LOS range of over $4 \mathrm{~km}$. The sensor provides measurements at a $20 \mathrm{~Hz}$ rate and with LOS velocity accuracy of $0.2 \mathrm{~cm} / \mathrm{s}$ and LOS range accuracy of $30 \mathrm{~cm}$. The velocity and range precision (as measurement on the prototype NDL flown onboard Morpheus in 2014) were measured to be $1.7 \mathrm{~cm} / \mathrm{sec}(1 \sigma)$ and $2.1 \mathrm{~m}(1 \sigma)$, respectively, which was dominated by the Morpheus vehicle vibration, acceleration, and angular motion during NDL signal acquisition.

\section{II.B. Lander Vision System}

The LVS consists of a Northrup Grumman LN200 Inertial Measurement Unit (IMU) and a visible wavelength monochrome camera rigidly mounted to a baseplate (See Figure 4). Processing for LVS is within the COBALT compute element, which hosts the LVS TRN algorithms and stores a reconnaissance map for image comparisons to determine map-relative position estimates. The LVS camera onboard COBALT is a Photon Focus global shutter 1024 x 1024 pixel CMOS (Complementary Metal-Oxide Semiconductor) monochrome camera with camera link interface and a Schneider ruggedized Cmount lens with a $60^{\circ} \mathrm{FOV}$.

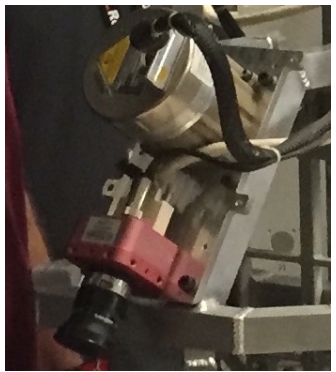

Figure 4. LVS camera and IMU.

\section{II.C. Compute Element}

The COBALT CE provides sensor data collection, payload timing, vehicle interfacing, data logging, telemetry and communications, and all the computing for the navigation filter state estimation, image processing, and 
vehicle pose initialization. The CE architecture is based on the ADAPT CE and includes an Aeroflex LEON3 compactPCI development board, an Alpha-Data Virtex-5 Field-Programmable Gate Array (FPGA), and an x86-based Single Board Computer (SBC) within a single 5-slot 6U compactPCI custom card cage. The FPGA captures and time-stamps payload sensor data and executes the image processing algorithms. The LEON3 board controls the FPGA and executes the COBALT navigation filter algorithms. The SBC logs all test telemetry, provides some algorithms computation, and maintains separate communication channels with the vehicle and the remote ground station. The SBC uses a second dedicated 100Base-T Ethernet port to send and receive continuous $50 \mathrm{~Hz}$ state updates between COBALT and Xodiac. The wireless radio is used to communicate with a remote base station approximately $1 \mathrm{~km}$ from the launch site. This link is used to initialize and control the payload and to monitor telemetry before and during flight.

\section{II.D. Payload Isolation}

The COBALT payload frame is enclosed to reduce potential electromagnetic interference (EMI) between COBALT and Xodiac. This enclosure also provides environmental (dust, moisture and thermal) protection; small fans are incorporated to ensure airflow during operation. Vibration isolators are incorporated between the payload frame and an internal base plate that holds the COBALT CE, power unit, and NDL electronics box. These isolators reduce vibration and shock to the payload during flight operations, as well as ground transport to and frame the launch/landing site. An onboard data recorder and three-axis accelerometer record the dynamic environment during transport and flight testing.

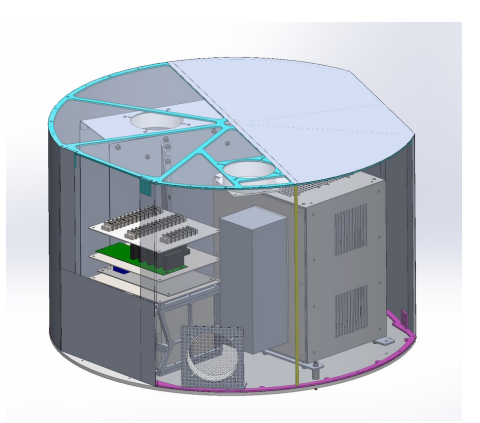

Figure 5. COBALT EMI Shroud.

\section{II.E. Power Unit}

The power system for COBALT has been upgraded from the original system flown on ADAPT while maintaining the same total mass. ADAPT used a custom lithium-polymer pack battery pack which was not removable from the payload assembly and had no capability for remote control or telemetry. COBALT leverages the power system developed for the SURROGATE ${ }^{19}$ and RoboSimian ${ }^{20}$ mobile robot programs. This system provides telemetry for the battery pack and remote control and telemetry for four individuallyswitched secondary loads. COBALT uses two BB-2590 batteries commercially available from Bren-tronics which can be removed from the payload and charged externally. The power capacity increased from $470 \mathrm{Wh}$ to $570 \mathrm{Wh}$ and maintaining the 90 -minute run-time capability.

\section{II.F. Xodiac Suborbital VTVL Vehicle}

The MSS XA-0.1E-5 (Xodiac) reusable, suborbital VTVL vehicle has been developed to service clients such as the NASA Flight Opportunities (FO) Program, which is funding the flight-test portion of the COBALT project. Figure 6 provides an illustration (left) of the Xodiac vehicle with payload frame, where the COBALT hardware will reside, and a photograph (right) of the first vehicle free flight conducted in January 2016. The Xodiac platform replaces the prior XA-0.1B (Xombie) vehicle, which previously completed eight client test programs for NASA-FO from 2011 to 2015, including flight tests for Draper Laboratories, Jet Propulsion Laboratory (with the ADAPT project), Astrobotic, and Carnegie Mellon University. The vehicle payload frame is designed for versatility, allowing clients to incorporate cameras, lasers, or other sensor systems within or external to the vehicle frame. The standard payload capacity is $50 \mathrm{~kg}$, and the vehicle is anticipated to achieve altitudes of up to $5 \mathrm{~km}$ and down-range profiles of up to $1 \mathrm{~km}$ (payload dependent). The vehicle has been undergoing envelope expansion flights in 2016, with the current flight tests achieving $449 \mathrm{~m}$ altitude and up to $27.6 \mathrm{~m} / \mathrm{s}$ velocity. 

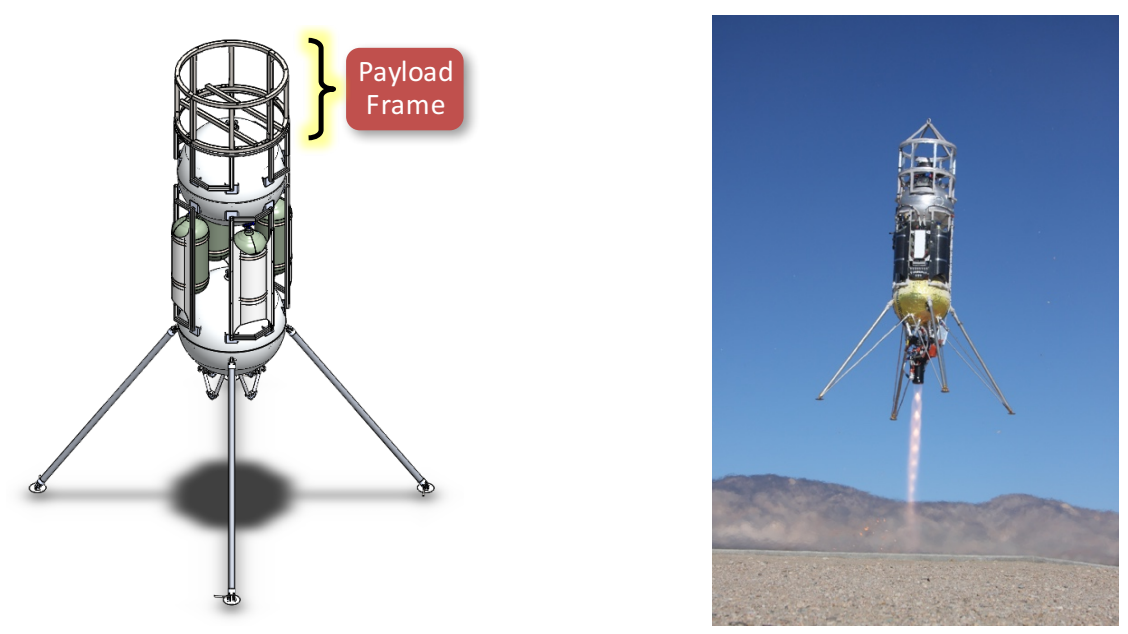

Figure 6. Xodiac Illustration and Free-Flight Image (courtesy of Masten).

Xodiac has been developed specifically to perform terrestrial, suborbital flight testing that emulates propulsive powered descent and soft touchdown phases of Mars or lunar landing. The platform enables lowrisk, closed-loop testing of payloads through the inclusion of software that accepts and monitors third-party GN\&C data. For the case of COBALT closed-loop flights, the Xodiac GPS-based navigation solution will provide a fall-back solution in the event that MSS onboard software detects anomalies with the COBALT navigation solution. The vehicle interfaces to the COBALT payload include an ethernet data connection and a Pulse-Per-Second (PPS) signal over RS422. The data connection is utilized for a $50 \mathrm{~Hz}$ packet between the vehicle and payload: the payload provides its navigation state at this rate, and the vehicle provides up-todate GPS time information from the vehicle, allowing COBALT to synchronize to GPS time and estimate the vehicle clock. The data connection and time synchronization also enable post-flight comparison of the COBALT navigation estimates versus the Xodiac differential-GPS-based navigation solution.

\section{Ground Integration and Testing}

\section{III.A. NDL implementation and functional testing}

The NDL implementation and functional verification was supported by a series of component-level and integrated-system tests to validate the sensor operation. Functional tests involve in-motion diffuse targets and static range targets, as well as tests to characterize the signal-to-noise ratio of the sensor. The in-motion targets include a belt sander and a treadmill that provide a Doppler shift to validate end-to-end functionality of the NDL components: transmitter laser to receiver to data acquisition system. This also provides an appropriate means for testing and debugging NDL signal processing algorithms. The static targets include a segmented and calibrated target board measuring $4.1 \mathrm{~m} \mathrm{x} 8.2 \mathrm{~m}$ that is set at approximately $250 \mathrm{~m}$ distance. The board includes segments with different reflectivity coefficients for radiometric measurements, as well as segments with varying range $(2.5 \mathrm{~cm}$ to $25 \mathrm{~cm}$ from the board back) for range precision and accuracy measurements. Other future NDL characterization tests will be performed to verify maximum operational range, as well as performance in representative thermal and vibration environments.

\section{III.B. Alignment and Calibration}

The LVS camera and IMU are attached to a payload sensor plate with alignment pins and metrology fiducials (ball nests), as seen in Figure 7 (left). A Leica metrology system is used to establish component-to-component alignment between the fiducials and pins. The COBALT navigation center coincides with the IMU navigation center, so the vendor IMU specification sheets are used to establish the IMU navigation center relative to the IMU alignment pins. The alignment of the camera requires a metrology calibration campaign (Figure 7, 
center and right). In this campaign, numerous images are taken of a high-contrast target board from various depths and covering the entire camera FOV, and metrology data is taken of the camera location relative to the target board locations. The data is used to produce a CAHVOR model for the camera, ${ }^{21}$ which is used by COBALT to transform image-based solutions into the COBALT navigation frame.
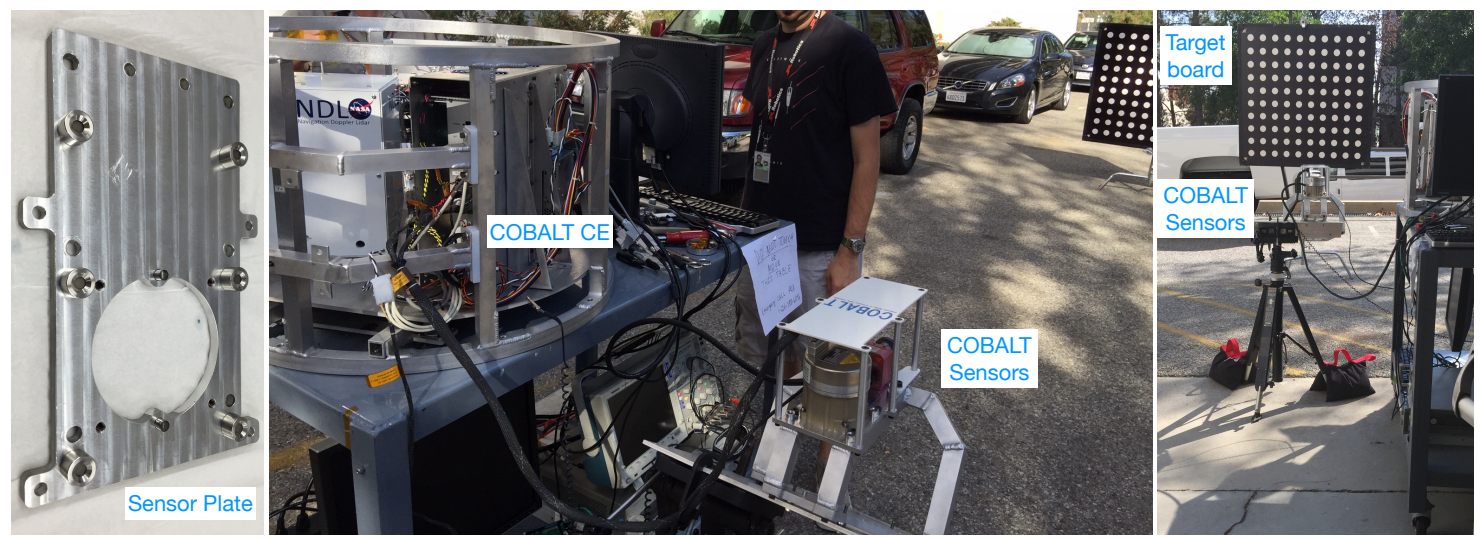

Figure 7. LVS sensor plate (left) and camera calibration (right)

Alignment of the NDL sensor into the COBALT navigation frame also requires a calibration and alignment campaign. The NDL optical head alignment is performed inline with methods established for the NDL during the ALHAT project. ${ }^{22}$ The calibration utilizes metrology measurements of NDL fiducial points on the base of the optical head, along with precise determination of the NDL LOS beams emanating from each of the three NDL telescopes. Alignment to COBALT is established through metrology measurements of the NDL optical-head fiducials relative to the COBALT payload sensor plate fiducials, which provides the chain of transforms to determine the NDL LOS beams relative to the COBALT navigation center.

Once the payload is delivered and integrated onto the Xodiac vehicle, metrology measurements will be used to align the COBALT sensor plate relative to alignment features on the Xodiac IMU, which is also the Xodiac navigation center. For the flight campaigns, the IMU-to-IMU alignment data will be used to transform the COBALT navigation state into the Xodiac navigation frame, which is a critical step for the closed-loop flight.

\section{III.C. FlatSat Interface Testing}

The FlatSat testing involves bench-top, static integration and functional testing between the COBALT CE and the NDL. The primary FlatSat objectives are to verify physical connections for electrical, power, and data, as well as software interfaces, commanding, telemetry, and data quality between the two subsystems. Figure 8 provides an image of a preliminary FlatSat activity between the NDL and a secondary COBALT $\mathrm{CE}$; this test was conducted during the final development work on the NDL at LaRC prior to shipment and integration with the full COBALT payload at JPL. Additional planned FlatSat objectives, following full systems integration, include mechanical and thermal checkouts, precision alignment (metrology) of the NDL to the COBALT IMU, and NDL operational tests to verify timing, data flow and telemetry. The NDL tests will be conducted with a belt sander to stimulate LOS velocity measurements.

\section{III.D. Xodiac and GPS Interface Testing}

An emulator of the Xodiac vehicle has been developed for interface testing of COBALT prior to delivery of the hardware for integration and testing with the actual Xodiac vehicle. This emulator captures all the channels to and from the vehicle, including a synthesized PPS and the ethernet messages. The emulator is based on a Digilent Zybo board with a Xilinx FPGA and an ARM Cortex-A9 processor running Linux. The emulator also features a Digilent Pmod GPS receiver to provide a PPS for testing, as well as independent position and velocity estimates, for precise timing synchronization to the COBALT payload and for use during dynamic 


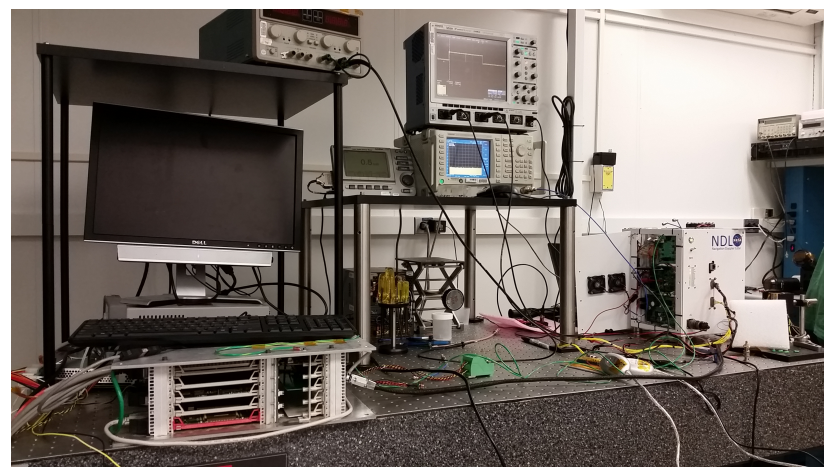

Figure 8. Preliminary FlatSat between secondary COBALT CE and NDL

testing at JPL. The emulator includes a Xodiac software simulator, which builds on ADAPT heritage, to generate flight-like data communication and verification. The emulator can also be used during initial interface testing with the Xodiac vehicle as an intermediary device to allow precise timing characterization.

\section{III.E. Functional Ground Testing}

Functional testing and partial validation of the COBALT payload will be conducted through a series of performance simulations and ground tests prior to the integration and testing of COBALT onboard Xodiac. Performance simulations of the COBALT estimator include reprocessing of ADAPT flight data (with synthetic NDL data), images rendered of the full COBALT trajectory, as well as static tests with real-time image processing using an image of Margaritifer Terra (a location on Mars). The simulations with ADAPT data and synthetic NDL data provide a means of testing and tuning the COBALT navigation filter prior to the actual Xodiac open-loop campaigns, which will then provide the final data for filter revisions prior to closed-loop flights. The real-time static tests are conducted in the COBALT lab (at JPL) and test the functionality of the COBALT CE to acquire and process camera, NDL and IMU measurements.

The ground tests will include a payload swing test and driving tests once the payload is fully integrated at JPL. A graphic depiction of the payload orientation is shown in Figure 9. The payload swing test will be conducted at JPL on a hangar crane, which provides a straightforward method for providing simple pendulum motion. This functional test will provide verification of the COBALT timing architecture, the phasing of NDL and IMU data, and the CE data handling and ground operations. The intent is to use dynamic motion to invoke measurement packets on both the NDL and IMU by post processing the measured sinusoidal motion of the payload. This will also be the first test of the NDL producing measurements with all three beams on the COBALT payload. No external truth measurements are needed, however LVS camera images are to be obtained for functionality and on board visual comparison.

To physically induce a sinusoidal motion observable by both the NDL and IMU, the entire payload including the sensors and avoincs is hung from a bridge crane using two $3 \mathrm{~m}$ long straps forming a 2-leg bridle hitch. This configuration allows for the payload to swing along a fixed axis with minimal yaw deviation. The payload is then raised to a height of $3 \mathrm{~m}$ and left to dwell to achieve a zero motion state. The system is then loaded to a $30^{\circ}$ off-nadir angle and released to swing achieving a period of approximately 3.5 seconds or $103^{\circ} / \mathrm{sec}$. Throughout the duration of the swing test, the payload runs on internal power.

The ground vehicle, driving tests will be conducted on a service All-Terrain Vehicle (ATV) with the payload mounted in a flight-like configuration toward the ground. The driving tests will provide further verification of NDL and IMU data phasing, as well as a functional dynamic test of the COBALT navigation filter using both NDL and IMU measurements. The filter solution is to be compared to GPS velocity obtained from the COBALT emulator. The sensor configuration shown in Figure 9 is designed to invoke velocity measurements on the NDL. The NDL LOS range is not intended to be used as it is less than the noise floor accuracy. Similarly, in this configuration TRN is not practical with this close proximity to the ground, however images will be taken in real-time at the nominal flight image rate to invoke the image 

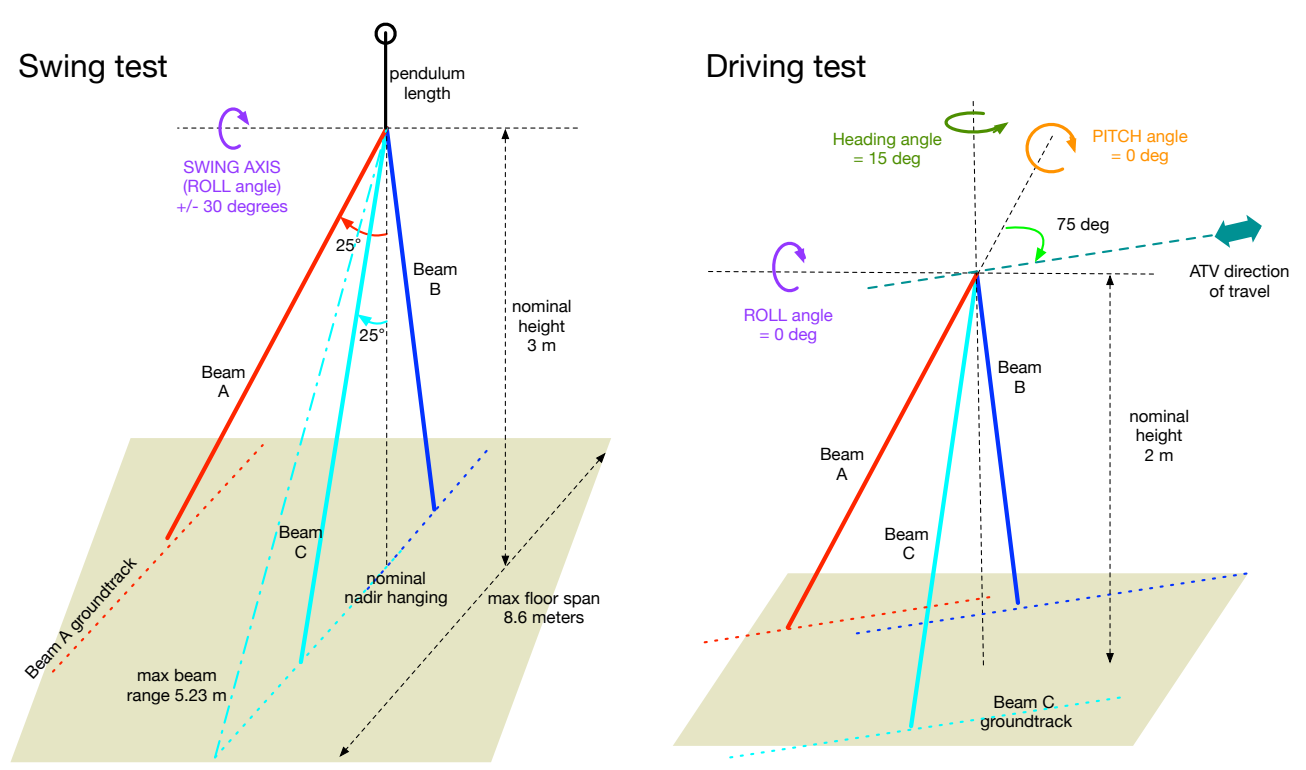

Figure 9. Illustrated depiction of the sensor configuration and motion directions during the swing tests (left) and driving tests (right)

processing and burden the COBALT CE procesing.

The complete payload is mounted to a base plate located on the bed of a Polaris Ranger XP 900 ATV. By mounting the payload at the edge of the bed, while offsetting the sensor assembly $15^{\circ}$ relative to the direction of travel, it is possible to invoke dynamic motion while avoiding beam interference with the ATV body. The ATV provides us with a minimum sensor height of $1.5 \mathrm{~m}$ and a velocity range of $0.1 \mathrm{~m} / \mathrm{s}$ to $10.0 \mathrm{~m} / \mathrm{s}$. During these tests the payload will be mounted on its flight isolation mounts and the vibration environment will be measured by independent sensors for characterization and analysis.

\section{Flight Campaign ConOps}

The ConOps for COBALT was developed to expand the flight test envelope for the NDL, compared to prior flight tests onboard the NASA Morpheus vehicle, ${ }^{7}$ and to test the performance of a precision navigation filter that incorporates both NDL and LVS measurements. The ConOps (Figure 10) also builds upon the prior ADAPT ConOps, with increases to altitude and vertical descent speed based on the new Xodiac vehicle performance capabilities with the COBALT payload.

The current planned envelope expansion flights for Xodiac will enable a maximum flight altitude of 527 $\mathrm{m}$ and a nominal flight time of 68 seconds from launch until the final $20 \mathrm{~m}$ altitude. The vehicle will perform a vertical ascent to the peak altitude, followed by a vertical descent to achieve a $25 \mathrm{~m} / \mathrm{s}$ downward velocity. At maximum downward velocity, a $300 \mathrm{~m}$ lateral divert maneuver will be planned onboard and executed to target a pre-determined landing location. During the final $20 \mathrm{~m}$ altitude, the Xodiac GN\&C system will auto-land the vehicle, which is a risk-reduction step with third-party payloads.

The COBALT navigation filter will ingest NDL measurements and LVS solutions whenever they are available during the flight profile. NDL velocity and range measurements will become available on ascent, above approximately $10 \mathrm{~m}$. The LVS TRN solutions will become available during ascent above 75-100 m. The COBALT navigation solution will be initialized on the ground, prior to launch, by using the Pose Initialization and Propagation (PIP) system developed for the ADAPT project. The PIP system uses the onboard camera to image GPS-surveyed ground targets and compute pre-launch vehicle pose. Prior to NDL measurements commencing, the COBALT navigation filter will propagate on the COBALT IMU. 

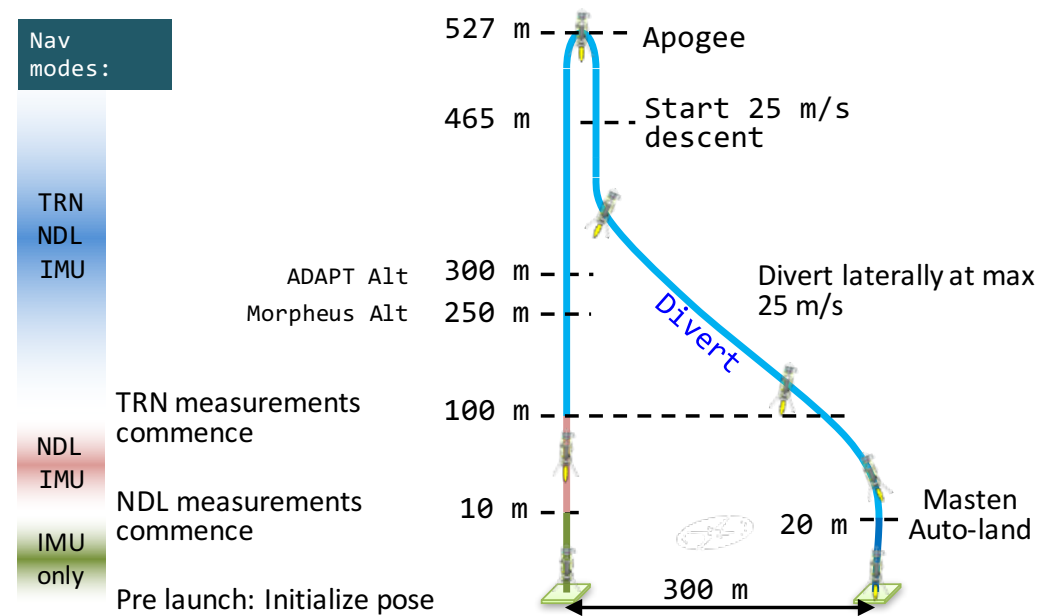

Figure 10. Illustration of COBALT flight profile and ConOps

\section{Status and Next Steps}

The GEN3 NDL is undergoing final stand-alone testing and software revisions prior to integration with the full COBALT payload hardware. Following full integration, the project will commence final FlatSat and Ground I\&T activities for final software revisions and preparation to integrate with the Xodiac vehicle. The vehicle itself will be conducting a final envelope expansion as part of a campaign with another FO payload; this flight will achieve the full altitude and lateral divert range planned for the COBALT ConOps. The openloop flight campaign is targeted for winter 2017, which will be followed by detailed data analysis, software revisions and a navigation filter model delivery to Masten in preparation for the closed-loop campaign in late FY2017. Following completion of the 2017 flight campaigns, the COBALT platform is targeted for future reuse in other PL\&HA technology development activities.

\section{Acknowledgments}

We want to acknowledge the large team of engineers across NASA at JSC, LaRC and JPL who are supporting the development, implementation and testing of COBALT. We also want to acknowledge the team at Masten that is working diligently to prepare the Xodiac vehicle for the COBALT flight campaigns. Additionally, the authors acknowledge the prior JPL ADAPT project team whose work the COBALT team is leveraging.

The COBALT Project derives funding and support from multiple NASA directorates and programs, including the Human Exploration and Operations Mission Directorate (HEOMD) Advanced Exploration Systems (AES) Program (through the Lander Technologies Project), the Space Technology Mission Directorate (STMD) Game Changing Development (GCD) Program, and the STMD Flight Opportunities (FO) Program. The LVS TRN software within COBALT has been developed through multi-directorate contributions, as well, including the NASA Science Mission Directorate (SMD) and STMD. As previously stated, the STMD-FO Program is provide the funding and coordination of the COBALT flight campaigns onboard the Masten Xodiac vehicle.

The work described herein is being performed within NASA JSC, LaRC and JPL, as well as within Masten Space Systems. The COBALT work at the Jet Propulsion Laboratory, California Institute of Technology, is being performed under contract with the National Aeronautics and Space Administration (Government sponsorship acknowledged). 


\section{References}

${ }^{1}$ Carson III, J. M., Seubert, C. R., Amzajerdian, F., Villalpando, C. Y., Bergh, C., O’Neal, T., Robertson, E. A., Hines, G. D., and Pierrottet, D. F., "COBALT: a Payload for Closed-Loop Flight Testing of ALHAT GN\&C Technologies on Terrestrial Rockets," Proc. AIAA Space 2016 Conference 86 Exposition, Long Beach, CA, September 2016.

${ }^{2}$ Epp, C. D., Robertson, E. A., and Carson III, J. M., "Developing Autonomous Precision Landing and Hazard Avoidance Technology from Concept through Flight-Tested Prototypes," Proc. AIAA GN\&C Conference, AIAA 2015-0324, Kissimmee, FL, Jan. 5-8 2015.

${ }^{3}$ Epp, C. and Smith, T., "The Autonomous Precision Landing and Hazard Detection and Avoidance Technology (ALHAT)," Proc. Space Technology and Applications International Forum (STAIF), 2007.

${ }^{4}$ Epp, C., Robertson, E., and Brady, T., "Autonomous Landing and Hazard Avoidance Technology (ALHAT)," Proc. IEEE Aerospace Conference (AEROCONF 2008), March 2008.

${ }^{5}$ Carson, J. M., Bailey, E. S., Trawny, N., Johnson, A. E., Roback, V. E., Amzajerdian, F., and Werner, R. A., "Operations Concept, Hardware Implementation and Ground-Test Verification of a Hazard Detection System for Autonomous and Safe Precision Lunar Landing," AAS/AIAA Astrodynamics Specialist Conference, AAS 13-856, Hilton Head, SC, Aug. 11-15 2013.

${ }^{6}$ Trawny, N., Huertas, A., Luna, M. E., Villalpando, C. Y., Martin, K., Carson, J. M., Johnson, A. E., Restrepo, C., and Roback, V. E., "Flight testing a Real-Time Hazard Detection System for Safe Lunar Landing on the Rocket-Powered Morpheus Vehicle," Proc. AIAA GNEC Conference, AIAA 2015-0326, Kissimmee, FL, Jan. 5-8 2015.

${ }^{7}$ Carson III, J. M., Robertson, E. A., Trawny, N., and Amzajerdian, F., "Flight Testing ALHAT Precision Landing Technologies Integrated Onboard the Morpheus Rocket Vehicle," Proc. AIAA Space 2015 Conference E Exposition, Pasadena, CA, August 2015.

${ }^{8}$ Trawny, N., Benito, J., Tweddle, B., Bergh, C. F., Khanoyan, G., Vaughan, G. M., Zheng, J. X., Villalpando, C. Y., Cheng, Y., Scharf, D. P., Fisher, C. D., Sulzen, P. M., Montgomery, J. F., Johnson, A. E., Aung, M., Regehr, M. W., Dueri, D., Açikmeşe, B., Masten, D., O'Neal, T., and Nietfeld, S., "Flight testing of terrain-relative navigation and large-divert guidance on a VTVL rocket," Proc. AIAA SPACE 2015 Conference \& Exposition, August 2015.

${ }^{9}$ Scharf, D., Regehr, M., Dueri, D., Acikmese, B., Vaughan, G., Benito, J., Ansari, H., Aung, M., Johnson, A., Masten, D., Nietfeld, S., Casoliva, J., and Mohan, S., "ADAPT Demonstrations of Onboard Large-Divert Guidance with a VTVL Rocket," Proc. IEEE Aerospace Conference, March 2014.

${ }^{10}$ Johnson, A., Bergh, C., Cheng, Y., et al., "Design and Ground Test Results for the Lander Vision System," $36^{\text {th }}$ Annual AAS Guidance and Control Conference, AAS 13-042, Breckenridge, CO, Feb. 1-6 2013.

${ }^{11}$ Johnson, A. E., Cheng, Y., Montgomery, J., Trawny, N., and Zheng, B. T. J., "Real-Time Terrain Relative Navigation Test Results from a Relevant Environment for Mars Landing," Proc. AIAA GNEC Conference, AIAA 2015-0851, Kissimmee, FL, Jan. 5-8 2015.

${ }^{12}$ Johnson, A. E., Cheng, Y., Montgomery, J., Trawny, N., Tweddle, B. E., and Zheng, J., "Design and Analysis of Map Relative Localization for Access to Hazardous Landing Sites on Mars," Proc. AIAA GNEC Conference, AIAA 2016-0379, San Diego, CA, Jan. 4-8 2016.

${ }^{13}$ Amzajerdian, F., Pierrottet, D., Petway, L., Hines, G., and Barnes, B., "Doppler lidar sensor for precision navigation in GPS-deprived environment," Proc. International Society for Optics and Photonics (SPIE), June 2013.

${ }^{14}$ Pierrottet, D. F., Amzajerdian, F., Petway, L. B., Hines, G. D., and Barnes, B., "Field Demonstration of a Precision Navigation Lidar System for Space Vehicles," Proc. AIAA Guidance, Navigation, and Control Conference, Boston, MA, August 2013.

${ }^{15}$ Amzajerdian, F., Pierrottet, D., Hines, G., Petway, L., and Barnes, B., "Fiber-based Doppler Lidar Sensor for Vector Velocity and Altitude Measurements," Frontiers in Optics 2015, OSA Technical Digest, 2015.

${ }^{16}$ Amzajerdian, F., Hines, G. D., Petway, L. B., Barnes, B. W., and Pierrottet, D. F., "Development and Demonstration of Navigation Doppler Lidar for Future Landing Mission," Proc. AIAA Space 2016 Conference 63 Exposition, Long Beach, CA, September 2016.

${ }^{17}$ Steering Committee for NASA Technology Roadmaps; National Research Council of the National Academies, NASA Space Technology Roadmaps and Priorities: Restoring NASA's Technological Edge and Paving the Way for a New Era in Space, The National Academies Press, 2012.

${ }^{18}$ Office of the Chief Technologist, 2015 NASA Technology Roadmaps, NASA, 2015.

${ }^{19}$ Hebert, P., Ma, J., Borders, J., Aydemir, A., Bajracharya, M., Hudson, H., Shankar, K., Karumanchi, S., Douillard, B., and Burdick, J., "Supervised Remote Robot with Guided Autonomy and Teleoperation (SURROGATE): A framework for whole-body manipulation," Proc. IEEE International Conference on Robotics and Automatic (ICRA), May 2015.

${ }^{20}$ Karumanchi, S., Edelberg, K., Baldwin, I., Nash, J., Reid, J., Bergh, C., Leichty, J., Carpenter, K., Shekels, M., Gildner, M., Newill-Smith, D., Carlton, J., Koehler, J., Dobreva, T., Frost, M., Hebert, P., Borders, J., Ma, J., Douillard, B., Backes, P., Kennedy, B., Satzinger, B., Lau, C., Byl, K., Shankar, K., and Burdick, J., "Team RoboSimian: Semi-autonomous Mobile Manipulation at the 2015 DARPA Robotics Challenge Finals," Journal of Field Robotics Special Issue: DARPA Robotics Challenge (DRC), 2015.

${ }^{21} \mathrm{Di}, \mathrm{K}$. and Li, R., "CAHVOR camera model and its photogrammetric conversion for planetary applications," Journal of Geophysical Research, Vol. 109, No. E4, April 2004.

${ }^{22}$ Carson III, J. M., Hirsh, R. L., Roback, V. E., Villalpando, C. Y., Busa, J. L., Pierrottet, D. F., Trawny, N., Martin, K. E., and Hines, G. D., "Interfacing and Verifying ALHAT Safe Precision Landing Systems with the Morpheus Vehicle," Proc. AIAA GNEC Conference, Kissimmee, FL, Jan. 5-8 2015. 